\title{
Antibody responses following induction of antigen-specific tolerance with antigen-coupled cells
}

Peschl, Patrick ; Reindl, Markus ; Schanda, Kathrin ; Sospedra, Mireia ; Martin, Roland ; Lutterotti, Andreas

\begin{abstract}
We have recently demonstrated the safety and tolerability of a novel therapeutic regimen employing autologous blood cells chemically coupled with seven myelin peptides to induce antigen-specific tolerance in MS (ETIMS study). The aim of the current study was an extended safety analysis to assess the effect of the ETIMS approach on antibodies to common autoantigens, the myelin peptides used and common recall antigens. None of the patients showed induction of autoantibody responses. One patient had a measurable myelin peptide-specific response at baseline, which was reduced after treatment. Total immunoglobulins and recall antibody responses showed no significant change.
\end{abstract}

DOI: https://doi.org/10.1177/1352458514549405

Posted at the Zurich Open Repository and Archive, University of Zurich

ZORA URL: https://doi.org/10.5167/uzh-99617

Journal Article

Accepted Version

Originally published at:

Peschl, Patrick; Reindl, Markus; Schanda, Kathrin; Sospedra, Mireia; Martin, Roland; Lutterotti, Andreas (2015). Antibody responses following induction of antigen-specific tolerance with antigen-coupled cells. Multiple Sclerosis, 21(5):651-655.

DOI: https://doi.org/10.1177/1352458514549405 
Antibody responses following induction of antigen-specific tolerance with antigen-coupled cells

Patrick Peschl ${ }^{1}$, Markus Reindl ${ }^{1}$, Kathrin Schanda ${ }^{1}$, Mireia Sospedra ${ }^{2}$, Roland Martin $^{2}$, Andreas Lutterotti ${ }^{1,2}$

${ }^{1}$ Clinical Department of Neurology, Innsbruck Medical University, Austria.

${ }^{2}$ Neuroimmunology and Multiple Sclerosis Research, Department of Neurology, University Hospital Zurich, Switzerland.

Corresponding author:

Andreas Lutterotti

Clinical Department of Neurology

Innsbruck Medical University

Anichstrasse 35

6020 Innsbruck, Austria

email: andreas.lutterotti@i-med.ac.at

Tel.: +43 51250482464

Fax.: +43 51250426312 


\section{Abstract}

We have recently demonstrated the safety and tolerability of a novel therapeutic regimen employing autologous blood cells chemically coupled with seven myelin peptides to induce antigen-specific tolerance in MS (ETIMS study). The aim of the current study was an extended safety analysis to assess the effect of the ETIMS approach on antibodies to common autoantigens, the myelin peptides used and common recall antigens. None of the patients showed induction of autoantibody responses. One patient had a measurable myelin peptide-specific response at baseline, which was reduced after treatment. Total immunoglobulins and recall antibody responses showed no significant change.

Keywords: multiple sclerosis, tolerance, antigen-coupled cell, antibody, myelin 


\section{Introduction}

MS is the most common disabling neurological disease in young adults. It is widely accepted that damages in the CNS results from a CD4 ${ }^{+}$-mediated autoimmune attack against myelin sheath associated antigens. ${ }^{1}$ We previously established an antigenspecific tolerance protocol that selectively targets these auto-reactive T-cells, employing autologous peripheral blood mononuclear cells (PBMC) pulsed with seven myelin peptides of myelin oligodendrocyte glycoprotein (MOG1-20, MOG3555), myelin basic protein (MBP13-32, MBP83-99, MBP111-129, MBP146-170) and proteolipid protein (PLP139-154) and chemically fixed with the cross linker EDC. We demonstrated the feasibility, safety and tolerability of a single infusion of chemically peptide-coupled PBMCs as tolerogenic vaccine in an open label, single center, dose escalation phase I trial (ETIMS trial). ${ }^{2}$ Furthermore, patients receiving more than $1 \times 10^{9}$ peptide-coupled cells showed a decrease in antigen-specific $\mathrm{T}$ cell responses. The aim of this study was to expand our safety analysis on ETIMS-treated patients analyzing the effect of antigen-coupled cells on the antibody responses against the target myelin peptides, common autoantigens, and recall antigens.

\section{Patients and Methods}

\section{Patients and serum samples}

The ETIMS trial was performed with approval of the ethics board of physicians of the University Hospital Hamburg-Eppendorf. ${ }^{2}$ In the dose escalation study, patients received a single infusion of autologous antigen-coupled cells at day 0 (individual doses shown in Table 1). Serum samples were taken from MS patients, one month before, three (patients \#1-\#7 and \#9) or six (patient \#8) months and 12 months (all patients) after treatment. All serum samples were stored at $-80^{\circ} \mathrm{C}$. Furthermore, all 
patients had a measurable $\mathrm{T}$ cell response to at least one of the peptides used in the trial, at baseline. ${ }^{2}$

\section{Antibody assays}

All serum samples were analyzed for IgG antibodies to native human MOG by a live

cell based immunoassay (CBA) as described before. ${ }^{3}$ Screening was done at 1:20 and 1:40 dilutions and samples were scored positive at a titer $>1: 160$.

Serum (1:100 diluted) lgG antibodies against the seven different peptides MOG1-20, MOG35-55, MBP13-32, MBP83-99, MBP111-129, MBP146-170 and PLP139-154 used in the ETIMS trial and against human MBP were analyzed by enzyme-linked immunosorbent assay (ELISA) as described before. ${ }^{4,5} \mathrm{~A}$ cut-off value of 0.5 optical density units was chosen to indicate positive antibody responses.

For the determination of rheumatoid factors (RF) and IgG antibodies against tetanus toxin, rubella, measles, anti-citrullinated peptide (CCP) and phospholipids commercially available ELISA kits from IBL International (Hamburg, Germany) were used. IgG antibodies to EBNA-1 and CMV were analyzed using ELISA kits from Euroimmun (Luebeck, Germany). Anti-nuclear antibody (ANA) were analyzed by indirect immunofluorescence (IIF) in HEp-20-10 cells using an IIF kit from Euroimmun. Serum samples were analyzed for total $\lg G$, IgM and IgA levels using a Beckman clinical nephelometer (Beckman Coulter).

\section{Statistical analysis}

Statistical analysis was performed using GraphPad Prism 6 (GraphPad, USA). 


\section{Results}

In this study we aimed to investigate antibody responses against myelin peptides, native human MOG, common autoantigens and recall antigens in patients treated with the ETIMS approach (Figure and Table 2). None of the patients had antibodies against native MOG, CCP, phospholipids and RF, whereas two patients had ANAs (patients \#2 and \#7) and two patients had anti-MBP antibodies (patients \#5 and \#7). In patient \#5 anti-MBP antibody levels were reduced ( $>2$-fold change) three and twelve months after treatment (Figure). Importantly, no new autoantibody responses were induced by the treatment (Figure and Table 2).

Only one patient (patient \#2) had measurable antibody reactivities against 8 of 9 myelin peptides used in the trial. These antibody responses were reduced ( $>2$-fold change) three and twelve months after treatment (Figure). However, this reduction was also seen for RF levels (>2-fold change).

Finally antibody responses to common recall antigens and total serum albumin and Ig levels were tested to address a peptide-specific effect of this therapy and to potentially exclude unspecific responses to the treatment. Overall antibody responses and Ig levels were not altered (>2-fold change) by the treatment (Figure, Table 2).

\section{Discussion}

In this extension study we analyzed the influence of the ETIMS approach on the antibody reactivity to common autoantigens, the myelin peptides used and common recall antigens. An important safety issue of antigen-specific tolerization is the induction of immune responses against the tolerizing antigen or even new autoantigens. Therefore, we were interested to asses changes in antibody responses to myelin peptides used for tolerization, native human MOG and MBP, other targets 
of autoimmunity responses and recall and control antibodies in any of the treated patients. As one important result of the safety analysis, we did not observe an increase in anti-myelin antibody reactivity, supporting the safety of the approach with regard to a potential induction of autoimmune responses as observed in previous tolerization strategies. ${ }^{6}$ Next we were interested to exclude potential induction of new autoimmunity by the treatment. Since antigen-coupled cells are known to undergo apoptosis after treatment ${ }^{2,7}$, there is a concern about induction of ANAs. In several autoimmune diseases, including lupus, mixed connective tissue disease and others, antibodies against nuclear antigens (e.g. ANA) are thought to be stimulated and sustained by apoptotic cells. In the current study we could not observe induction of ANAs in any of the patients. Likewise, we did not detect an induction of anti-CCP, anti-phospholipid antibodies or RF in any of the patients.

We found a positive antibody response to $8 / 9$ myelin peptides in one single patient (\#2) at baseline. Since this patient was negative for antibodies to native MOG it is very unlikely that these peptide-specific antibodies have a pathogenic role, although they still might reflect tissue damage and ongoing neuorinflammation. ${ }^{3,8-10}$ There was a clear reduction in myelin peptide specific antibody responses following ETIMS treatment. However, we have also observed a reduction of RF and anti-rubella antibodies thus challenging the specificity of the effect.

In summary the extended safety analysis of the ETIMS phase I study does not suggest an increase in autoantibodies or induction of new autoantibody reactivities in MS patients treated with the ETIMS approach. The results underscore the safety of this novel antigen-specific therapy.

\section{Disclosure of conflicts of interest}


A Lutterotti and R Martin are listed as co-inventors on a University Zürich patent related to the use of antigen-coupled cells in MS.

\section{Acknowledgements}

The authors thank Ingrid Gstrein for the measurement of total albumin and Ig levels and all ETIMS investigators . 


\section{References}

1. Sospedra M and Martin R. Immunology of multiple sclerosis. Annu Rev Immunol. 2005; 23: 683-747.

2. Lutterotti A, Yousef S, Sputtek A, et al. Antigen-specific tolerance by autologous myelin peptide-coupled cells: a phase 1 trial in multiple sclerosis. Sci Transl Med. 2013; 5: 188ra75.

3. Di Pauli F, Mader S, Rostasy K, et al. Temporal dynamics of anti-MOG antibodies in CNS demyelinating diseases. Clin Immunol. 2011; 138: 247-54.

4. Pittock SJ, Berger T, Bruck W, et al. Anti-myelin antibodies: Frequency, stability and clinicopathologic associations in a biopsy MS cohort. Mult Scler. 2005; 11: $109-$

5. Khalil M, Reindl M, Lutterotti A, et al. Epitope specificity of serum antibodies directed against the extracellular domain of myelin oligodendrocyte glycoprotein: influence of relapses and immunomodulatory treatments. . J Neuroimmunol. 2006; 174: $147-56$.

6. Bielekova B, Goodwin B, Richert N, et al. Encephalitogenic potential of the myelin basic protein peptide (amino acids 83-99) in multiple sclerosis: results of a phase II clinical trial with an altered peptide ligand. Nat Med. 2000; 6: 1167-75. 7. Turley DM and Miller SD. Peripheral tolerance induction using ethylenecarbodiimide-fixed APCs uses both direct and indirect mechanisms of antigen presentation for prevention of experimental autoimmune encephalomyelitis. $J$ Immunol. 2007; 178: 2212-20.

8. Krumbholz M, Derfuss T, Hohlfeld R and Meinl E. B cells and antibodies in multiple sclerosis pathogenesis and therapy. Nat Rev Neurol. 2012; 8: 613-23. 
9. Quintana FJ, Farez MF, Viglietta V, et al. Antigen microarrays identify unique serum autoantibody signatures in clinical and pathologic subtypes of multiple sclerosis. Proc Natl Acad Sci USA. 2008; 105: 18889-94.

10. Robinson WH, Fontoura P, Lee BJ, et al. Protein microarrays guide tolerizing DNA vaccine treatment of autoimmune encephalomyelitis. Nat Biotechnol. 2003; 21: 1033-9. 
Figure: Effect of ETIMS treatment on antibody levels to common autoantigens, the petides used for tolerance induction and common recall antigens. Antibody responses to myelin peptides and MBP were measured by ELISA, whereas antibodies to native human MOG were measured by CBA. Serum antibodies to recall antigens (tetanus, measles and rubella), common viruses (EBV and CMV), ANA, CCP, RF and phospholipids were measured by ELISA and ANA were measured by indirect immunofluorescence. Graphs show the individual antibody levels before therapy and 3/6 and 12 months after ETIMS treatment. Cut-off values are indicated by the dashed lines. Values are shown as antibody titers, antibody concentration in $\mathrm{IU} / \mathrm{ml}$, OD units or $\mathrm{mg} / \mathrm{dl}$. 

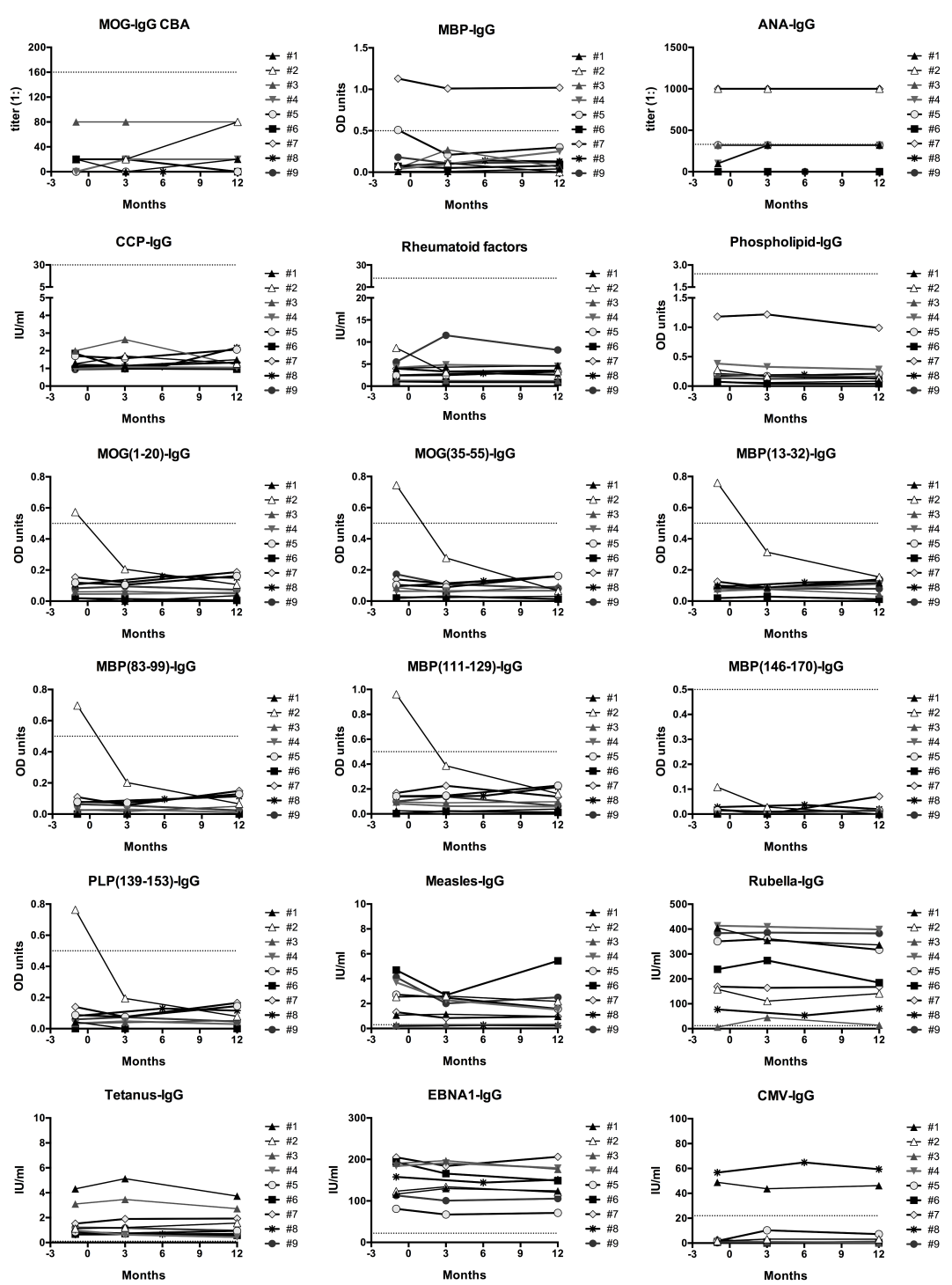
Table 1. Clinical data and treatment regimens of patients.

\begin{tabular}{|c|c|c|c|c|c|c|}
\hline Patients & Cell dose $^{1}$ & Age & Sex & MS & Duration $^{2}$ & EDSS \\
\hline$\# 1$ & $1 \times 10^{3}$ & 49 & $f$ & SP & 5 & 3.5 \\
\hline$\# 2$ & $1 \times 10^{5}$ & 39 & $f$ & $\mathrm{RR}$ & 5 & 1.5 \\
\hline \# 3 & $1 \times 10^{7}$ & 27 & $f$ & $\mathrm{RR}$ & 2 & 1 \\
\hline$\# 4$ & $1 \times 10^{8}$ & 42 & $f$ & $\mathrm{RR}$ & 2 & 1.5 \\
\hline \# 5 & $5 \times 10^{8}$ & 44 & $f$ & RR & 16 & 4 \\
\hline$\# 6$ & $1 \times 10^{9}$ & 43 & $f$ & SP & 13 & 4.5 \\
\hline$\# 7$ & $1 \times 10^{9}$ & 34 & $f$ & RR & 2 & 1.5 \\
\hline$\# 8$ & $2.5 \times 10^{9}$ & 42 & $f$ & RR & 4 & 1.5 \\
\hline \#9 & $3 \times 10^{9}$ & 42 & $\mathrm{~m}$ & RR & 3 & 2 \\
\hline
\end{tabular}

1) Cell dose is given as the number of antigen-coupled cells infused; 2) duration of disease in years. f: female; m: male; MS: multiple sclerosis; SP: secondary-progressive; RR: relapsing-remitting; EDSS: expanded disability status scale 
Table 2. Antibody and serum protein levels after ETIMS treatments.

\begin{tabular}{|c|c|c|c|c|}
\hline Antigen & -1 month & $+3 / 6$ months & +12 months & P-value \\
\hline \multicolumn{5}{|l|}{ Autoantibodies } \\
\hline MOG-IgG CBA & $0(0 \%)$ & $0(0 \%)$ & $0(0 \%)$ & $1.000^{1}$ \\
\hline MBP-IgG & $2(22 \%)$ & $1(11 \%)$ & $1(11 \%)$ & $0.746^{1}$ \\
\hline ANA-lgG & $2(22 \%)$ & $2(22 \%)$ & $2(22 \%)$ & $1.000^{1}$ \\
\hline CCP-IgG & $0(0 \%)$ & $0(0 \%)$ & $0(0 \%)$ & $1.000^{1}$ \\
\hline Rheumatoid factor & $0(0 \%)$ & $0(0 \%)$ & $0(0 \%)$ & $1.000^{1}$ \\
\hline Phospholipid-lgG & $0(0 \%)$ & $0(0 \%)$ & $0(0 \%)$ & $1.000^{1}$ \\
\hline \multicolumn{5}{|c|}{ Anti-peptide antibodies } \\
\hline MOG(1-20)-IgG & $1(11 \%)$ & $0(0 \%)$ & $0(0 \%)$ & $0.354^{1}$ \\
\hline MOG(35-55)-IgG & $1(11 \%)$ & $0(0 \%)$ & $0(0 \%)$ & $0.354^{1}$ \\
\hline MBP(13-32)-IgG & $1(11 \%)$ & $0(0 \%)$ & $0(0 \%)$ & $0.354^{1}$ \\
\hline MBP(83-99)-lgG & $1(11 \%)$ & $0(0 \%)$ & $0(0 \%)$ & $0.354^{1}$ \\
\hline MBP(111-129)-IgG & $1(11 \%)$ & $0(0 \%)$ & $0(0 \%)$ & $0.354^{1}$ \\
\hline MBP(146-170)-IgG & $0(0 \%)$ & $0(0 \%)$ & $0(0 \%)$ & $1.000^{1}$ \\
\hline
\end{tabular}


PLP(139-153)-IgG

$1(11 \%)$

$0(0 \%)$

$0(0 \%)$

$0.354^{1}$

Anti-viral and vaccine antibodies (common recall antigens)

\begin{tabular}{|c|c|c|c|c|}
\hline Measles-lgG & $9(100 \%)$ & $9(100 \%)$ & $9(100 \%)$ & $1.000^{1}$ \\
\hline Rubella-IgG & $8(89 \%)$ & $9(100 \%)$ & $9(100 \%)$ & $0.354^{1}$ \\
\hline Tetanus-IgG & $9(100 \%)$ & $9(100 \%)$ & $9(100 \%)$ & $1.000^{1}$ \\
\hline EBNA1-lgG & $9(100 \%)$ & $9(100 \%)$ & $9(100 \%)$ & $1.000^{1}$ \\
\hline CMV-IgG & $2(22 \%)$ & $2(22 \%)$ & $2(22 \%)$ & $1.000^{1}$ \\
\hline
\end{tabular}

\section{Serum proteins}

Albumin (mg/dl) $4277(263)$ $4374(204)$ $4233(211)$

$0.313^{2}$

Total-IgG (mg/dl) ${ }^{3}$ 1059 (256) 1061 (223) 1055 (235 ) $0.362^{2}$

Total-IgM (mg/dl) ${ }^{3}$ 148 (49) 149 (223) 149 (53) $0.971^{2}$

Total-lgA (mg/dl) ${ }^{3}$ 273 (173)

266 (161)

270 (177)

$0.701^{2}$

${ }^{1}$ significance of group differences analyzed by Chi-Square test or $^{2}$ repeated-measures one-way ANOVA, ${ }^{3}$ data are shown as mean (standard deviation). 